\title{
Efectos del choque petrolero de 20|4-20 I5 en la captación de divisas y el gasto público
}

\author{
Effects of 20 I4-20I5 Oil Shock on Foreign Currency Flows and Public Spending
}

\begin{abstract}
Resumen
Se presentan las condiciones de sobreoferta de hidrocarburos y de desaceleración de la economía mundial en que se dio la caída de los precios del petróleo entre mediados de 2014 y principios de 2015. Se hace una cuantificación de los efectos directos de lo anterior en la cantidad de divisas que recibe el país y en los ingresos del sector público, en el marco de una reforma energética que apenas en este año empezaría a operar. Se analiza la decisión de aplicar un recorte de 124300 millones de pesos en el presupuesto de egresos para 2015, de los que $70 \%$ corresponde a gasto de inversión, a pesar de contarse con dos contenciones: las coberturas petroleras, contratadas con suficiente anticipación, y la reforma hacendaria, que en 2014 permitió aumentar en 1.3\% respecto al PIB los ingresos tributarios no petroleros, $\mathrm{CO}^{-}$ eficiente que seguirá creciendo hasta 2018. La forma en que se llevaron a cabo los ajustes y el monto de los recursos sacrificados sugiere que el gobierno federal encontró en el entorno petrolero un justificante para generar ahorros y no permitir que siga creciendo, en un año de elecciones, el déficit del sector público, que en 2014 fue el mayor del siglo xxI, aunque ello impactará el crecimiento de corto y mediano plazo de la economía.
\end{abstract}

Palabras clave:

- Hidrocarburos

- Tipos de cambio

- Gasto público

\section{Abstract}

Roberto Gutiérrez R.*

The oversupply of hydrocarbons and the slowdown of the global economy are presented as for their effect on the collapse of oil prices between mid-2014 and early 2015 . Quantification is made of the direct effects of the above in the amount of foreign currency received by the country as well as in the public sector revenues, having as a background an energy reform to start being implemented in this year. Then the decision to apply a budget cut of 124300 million pesos for 2015 , of which $70 \%$ are investment spending, is analyzed taking into consideration two previous instruments that could have prevented such a decision to be made: oil hedges, acquired with enough anticipation, and the tax reform that allowed 1.3\% increase in non-oil tax revenues relative to GDP in 2014, ratio that will keep growing until 2018. The way adjustments were conducted and the amount of slaughtered resources suggests that the federal government found in the oil environment a way to generate savings and justify putting a halt, in an election year, to the public sector deficit which in 2014 was the largest in the XxI century, however its impact on economic growth in the short and medium term.

\section{Keywords: \\ - Hydrocarbons \\ - Foreign Exchange \\ - Public Economics}

JEL: L71, F31, H5

\section{Introducción}

El 30 de enero de 2015 la Secretaría de Hacienda y Crédito Público (SHCP) anunció un recorte de 124300 millones de pesos en los egresos del gobierno federal presupuestados para ejercerse durante el año. La cantidad equivale a $0.7 \%$ del producto interno bruto (PIB) y contablemente es consecuente con la reducción del precio del crudo mexicano de exportación. Empero, la me-

\footnotetext{
* Profesor-Investigador, Departamento de Economía, Universidad Autónoma Metropolitana, Iztapalapa.
} robertogtz@yahoo.com. 
dida se llevó a cabo no obstante que durante 2014 el gobierno federal contó con ingresos tributarios no petroleros superiores en 1.3\% del PIB respecto a los obtenidos en 2013, los cuales se espera suban hasta al menos 3\% al final del sexenio, en virtud de la reforma hacendaria aprobada por el Congreso en noviembre de 2013 y las nuevas medidas recaudatorias del Sistema de Administración Tributaria (SAT). Además, a fines de 2014 adquirió coberturas petroleras que le costaron más de 10 mil millones de pesos y le permiten restituir al erario $57.3 \%$ de la pérdida de recursos por la reducción de precios del energético, menos el costo de la prima.

El trabajo analiza las condiciones en que se ha dado la caída de los precios, qué impacto tiene ésta en la cantidad de divisas que ingresa al país, en qué consisten las coberturas petroleras, de qué manera actúan como paliativo para la reducción de los ingresos presupuestales del gobierno federal y a qué reordenamiento de las finanzas públicas da lugar todo esto, particularmente en términos del déficit y de la deuda externa, que a partir de 2015 va a estar afectada por un previsible incremento de la tasa de interés en Estados Unidos (eu). Dado que el recorte al gasto público presupuestal se llevó a cabo sin reducir el efecto de los ingresos esperados por las coberturas, se observa, sin duda, que el gobierno federal ha encontrado en el entorno una oportunidad para generar ahorros que le permitan, en un año de elecciones, no presionar más el déficit del sector público, cuyo nivel en 2014 fue el más alto en lo que va del siglo, $3.6 \%$ del PIB, en tanto los requerimientos financieros del sector público (RFSP) llegaron a 4.2 por ciento.

Desafortunadamente, el recorte impacta de manera negativa la capacidad de gasto de muchas familias y empresas y, al cancelar múltiples proyectos de inversión, pone en entredicho el crecimiento de la economía para 2015. Esto, aunado a las malas perspectivas de recepción de inversiones en la industria de los hidrocarburos que se proyectaban a partir de la puesta en operación de la reforma energética constitucional de 2013 y sus leyes secundarias y reglamentos de 2014, desvanece la posibilidad de que en este sexenio la economía crezca a una tasas promedio más elevada (se pronosticaba por lo menos el doble) de la observada entre 2001 y 2012 (2.4 por ciento).

\section{La caída de los precios}

Entre junio de 2014 y febrero de 2015 los precios internacionales del petróleo experimentaron un descenso de $60 \%$, no del todo sorpresivo en virtud de que la economía europea casi entró en una triple recesión en 2014; se desaceleró la 
economía china; se profundizó la así llamada revolución del gas y petróleo shale (de lutitas o pizarra) en Eu, y se exacerbaron las pujas de los tres productores más grandes del mundo - Arabia Saudita, EU y Rusia- por aumentar su autosuficiencia energética y/o acrecentar su participación de mercado, cercana en conjunto a 40\%. ${ }^{1}$ De esta manera, el precio de la mezcla mexicana bajó de 98.79 dólares por barril (d/b) en junio de 2014 (intradía llegó a ubicarse en hasta $135 \mathrm{~d} / \mathrm{b}$ ) a $39.26 \mathrm{~d} / \mathrm{b}$ promedio en enero de 2015 , como muestra la gráfica 1.

\section{Gráfica I}

Precio mensual mezcla crudo mexicano de exportación Ene, 2012-Ene, 2015 Dls/barril

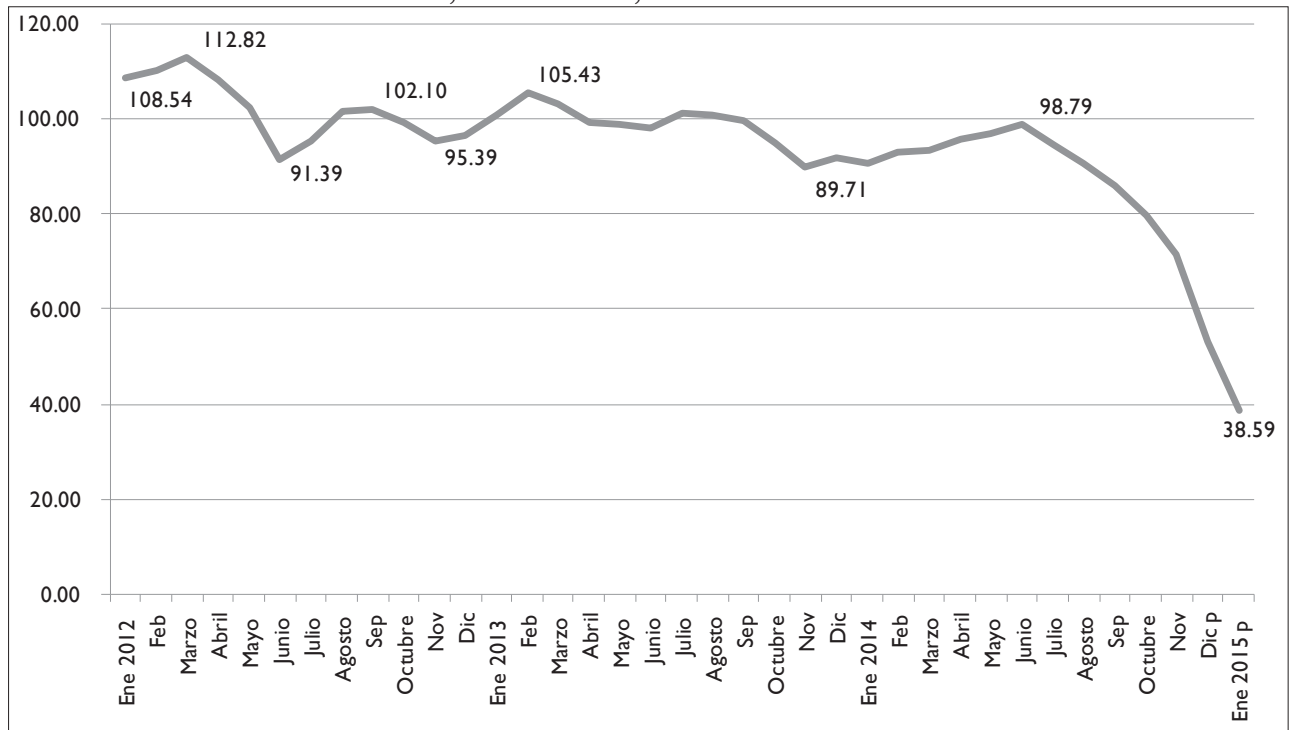

Fuente: Pemex, Indicadores Petroleros, diferentes números.

\footnotetext{
${ }^{1}$ La prioridad para EU es que se hagan efectivas las sanciones económicas de occidente a Rusia por haberse adjudicado, en marzo de 2014, la República Autónoma de Crimea, legalmente controlada por Ucrania. Para ello se sirve de su producción creciente de petróleo y gas shale, con lo que debilita los precios de sus dos principales productos de exportación. Mientras tanto, Arabia Saudita busca dos cosas: por una parte, quebrar la producción de petróleo y gas shale de Eu, culpable según su narrativa de la sobreoferta mundial de petróleo (alrededor de 2 millones de barriles diarios, mmbd), para lo que se ha propuesto no sacrificar su producción de crudo y lograr que sus socios del Golfo, Kuwait, Emiratos Árabes Unidos y Qatar, tampoco lo hagan, ya que el grupo tiene poder monopólico en la Organización de Países Exportadores de Petróleo (OPEP), al ser responsable de más de la mitad de la producción de ésta. Ello lo asocia con la estimación de que el umbral de costos de producción de gas y petróleo shale en Eu es de entre 40 y 60 d/b de petróleo crudo equivalente, mientras en el Golfo es de $6 \mathrm{~d} / \mathrm{b}$. Por otra parte busca presionar a Rusia, con una economía debilitada, para que retire su apoyo al gobierno de Siria.
} 
Es evidente que el precio del petróleo es excesivamente volátil; por ejemplo, en los 20 años previos a 2015 se movió de un promedio anual piso de 10.17 d/b en 1998 a un techo de $101.97 \mathrm{~d} / \mathrm{b}$ en 2012, para caer de nuevo a $39.26 \mathrm{~d} / \mathrm{b}$ en 2015 (véanse valores de las barras de la gráfica 2, a leerse en el eje izquierdo). En términos de variaciones porcentuales, las cuales se asocian lo mismo a choques de demanda - principalmente caída del consumo, la inversión y/o las exportaciones-, que de oferta (conflictos bélicos de los países productores, caída de reservas probadas, desastres naturales, etc.), las recesiones de 1997 del Sureste Asiático y de 1998 de Rusia provocaron que la mezcla mexicana se redujera en dos años 46\%; la "burbuja tecnológica" de 2001 condujo a una caída de 25\%; la así llamada Gran Recesión de 2009 llevó a un descenso de $31.9 \%$, y los acontecimientos de 2014 propiciaron una disminución de 54.9\%, como muestra la línea continua de la gráfica 2, a leerse en el eje derecho.

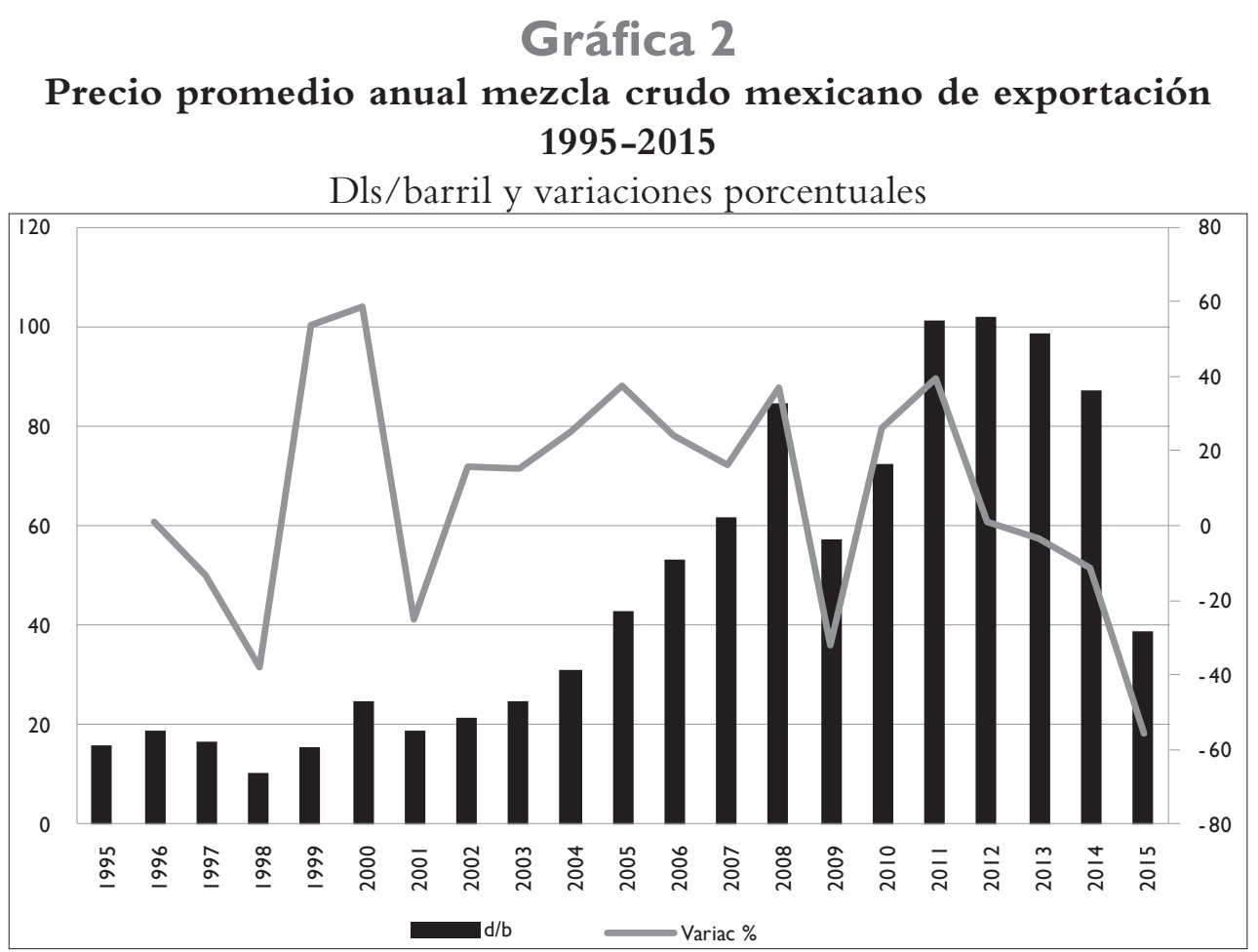

Fuente: Pemex, Anuario Estadístico e Indicadores Petroleros, diferentes números.

Lo que cuesta mucho trabajo a los seguidores del mercado petrolero internacional reconocer, particularmente los responsables de las finanzas públicas, es que la caída de los precios siempre ha estado precedida de períodos de creci- 
miento estable de la economía mundial, como la década de los noventa, los siete años posteriores a 2001, y el lapso de recuperación de 2010-2013, posterior a la Gran Recesión. Además, haber actuado sobre el gasto público parecía contradictorio con la realidad: la reacción de los precios del petróleo después de una caída pronunciada tiende a ser muy fuerte, como evidencia la gráfica 2 (años de 1999, 2002 y 2010-2011); a fines de enero de 2015 los mercados empezaron a marcar el piso a la caída más reciente de precios (los $39.26 \mathrm{~d} / \mathrm{b}$ de la gráfica 1 parecen ser una cota inferior); el gobierno contaba con recursos adicionales crecientes gracias a la puesta en operación de la reforma hacendaria (1.3 puntos porcentuales más de ingresos tributarios respecto al PIB captados en 2014), y por primera vez desde 2009 recibiría recursos, seguramente considerables, por concepto de las coberturas petroleras que había contratado. Además, sus importaciones de gasolinas y diesel se abaratarían, sin que por ello tuviera que modificar los precios al menudeo de dichos carburantes.

\section{Balanza petrolera}

La balanza petrolera de México, que por ley fue hasta 1914 la de Pemex, está compuesta, en cuanto a las exportaciones, por la venta de crudo, productos petrolíferos (incluyendo gas y condensados) y productos petroquímicos, sobre todo primarios. Respecto a las importaciones, se compone de productos petrolíferos (sobre todo gasolina y diesel), gas natural y licuado, y productos petroquímicos tanto primarios (en especial gas natural) como secundarios importados por Pemex. Es decir, existe cierto grado de comercio intraindustrial, ya que por ejemplo Pemex importa y al mismo tiempo exporta gasolinas, diesel y gas natural. Es claro que la puesta en operación de la reforma energética cambiará la práctica de depender de las operaciones de Pemex en este campo, ya que las empresas privadas podrán importar la mayor parte de los insumos y carburantes que requieran para sus actividades de producción y ventas al menudeo.

Como muestra el cuadro 1, el valor de las exportaciones de Pemex alcanzó su tope en 2011, cuando se colocaron en el exterior casi 60000 millones de dólares de crudo y petrolíferos (las de petroquímicos han sido marginales). Todavía en 2014, de acuerdo con cifras preliminares, dichas exportaciones fueron de 41524 millones de dólares. Pero como las importaciones de gas natural seco y petrolíferos han aumentado a lo largo del tiempo, con lo que el total de importaciones de Pemex se quintuplicó entre 2004 (5 565 millones de dólares) y 2014 (28 652 millones de dólares), el saldo comercial de dicha 
industria ha tendido a reducirse: de un pico de 29054 millones de dólares en 2011 pasó a sólo 12872 millones en 2014. Asimismo, se observa que en 2014 el saldo positivo de petróleo crudo no fue capaz de financiar ni la mitad del saldo negativo de productos petrolíferos. De no recuperarse los precios del crudo, el problema va a continuar, a menos que se inicie de manera decidida una política de diversificación basada en energéticos no convencionales, la cual ha sido postergada desde los años ochenta del siglo xx.

\section{Cuadro I}

\section{Balanza de divisas de la industria petrolera de México 2000-2014}

Millones de dólares

\begin{tabular}{|c|c|c|c|c|c|c|c|c|c|c|c|}
\hline Concepto & 2000 & 2002 & 2004 & 2006 & 2008 & 2009 & 2010 & 2011 & 2012 & 2013 & 2014 \\
\hline Exportaciones & 15966.3 & 14691.1 & 23421.6 & 38671.9 & 49543.3 & 30513.6 & 41025.8 & 59858.9 & 52075.3 & 48593.8 & 41524.1 \\
\hline Crudo & 14552.9 & 13392.2 & 21257.9 & 34706.8 & 43341.5 & 25605.4 & 35985.4 & 49379.6 & 46852.4 & 42723.2 & 35931.2 \\
\hline Petrolíferos 1/ & 1166.7 & 1186 & 1947 & 3666.4 & 5853.2 & 4760.9 & 4795.8 & 10219.1 & 4939.9 & 5699.6 & 5444.7 \\
\hline Petroquímicos & 246.7 & 112.9 & 216.7 & 298.7 & 348.6 & 147.3 & 244.6 & 260.2 & 283 & 171 & 148.2 \\
\hline Importaciones & 4671.9 & 3316.5 & 5565.1 & 11291.5 & 23474.1 & 14108.7 & 21448.8 & 30804.6 & 31098.9 & 28330.4 & 28651.6 \\
\hline Gas natural seco & 366.5 & 775.4 & 1715.1 & 1134.5 & 1423.6 & 632.8 & 939.2 & 1272.2 & 1216.2 & 2495.3 & 2855.7 \\
\hline Petrolíferos & 4233.4 & 2495.2 & 3791.6 & 10028.8 & 21892.8 & 13307.8 & 20335.4 & 29407.6 & 29630.9 & 25706.2 & 25701.4 \\
\hline Petroquímicos & 72 & 45.9 & 58.4 & 128.2 & 157.7 & 168.1 & 174.2 & 124.7 & 251.8 & 128.9 & 94.5 \\
\hline Saldo & 11294.4 & 11374.6 & 17856.5 & 27380.4 & 26069.2 & 16404.9 & 19577 & 29054.3 & 20976.4 & 20263.4 & 12872.5 \\
\hline Crudo & 14552.9 & 13392.2 & 21527.9 & 34706.8 & 43341.5 & 25605.4 & 35985.4 & 49379.6 & 46852.4 & 42723.2 & 35931.2 \\
\hline Gas natural seco & -366.5 & -775.4 & -1715.1 & -1134.5 & -1423.6 & -632.8 & -939.2 & -1272.2 & -1216.2 & -2495.3 & -2855.7 \\
\hline Petrolíferos & -3066.7 & -1309.2 & -1844.6 & -6362.4 & -16039.6 & -8546.9 & -15539.6 & -19188.5 & -24691 & -20006.6 & -20256.7 \\
\hline Petroquímicos & 174.7 & 67 & 158.3 & 170.5 & 190.9 & -20.8 & 70.4 & 135.5 & 31.2 & 42.1 & 53.7 \\
\hline
\end{tabular}

Haciendo previsiones, si el precio del crudo mexicano de exportación permanece durante 2015 en un promedio inferior a $40 \mathrm{~d} / \mathrm{b}$, las exportaciones totales de la industria bajarían a unos 20000 millones de dólares; las importaciones podrían ubicarse en 25000 millones -máxime si el Departamento de Comercio de Eu autoriza que México compre a dicho país $100 \mathrm{mbd}$ de crudo ligero para reforzar la mezcla que envía a sus refinerías-, y la balanza petrolera experimentaría un déficit cercano a 5000 millones de dólares. Esta sería una situación no vista desde la primera mitad de la década de los setenta, previa la era Cantarell, cuando la balanza de la industria petrolera fue negativa. 
En caso de que se materialicen las tendencias anteriores, la participación de las exportaciones de Pemex en las exportaciones totales de México continuaría disminuyendo: $72.9 \%$ en $1982,18.3 \%$ en 2002, 14\% en 2012 y 5\% en 2015 (ya en 1998 estuvo en 6.1\%). Por su parte, el déficit de la balanza comercial del país, que en 2014 fue de 2441 millones de dólares, podría llegar a casi 15000 millones de dólares en 2015. Debido a que existen serias limitaciones para que éste se compense con inversión extranjera directa (IED) en la industria de los hidrocarburos, dado que a nivel mundial 2015 será un año de transición para la industria, al tiempo que habrá volatilidad en los mercados de capital en cuanto el Banco de la Reserva Federal (FED) de Eu decrete el alza de las tasas de interés, se prevé también una contracción del flujo neto de inversión de cartera hacia México. Con ello, el peso mexicano podría mantenerse lateral en 2015 y la balanza de pagos enfrentar desequilibrios no vistos desde 2009, año de recesión. Estas previsiones, más que la sola caída de los precios del petróleo, explican el pesimismo del Banco de México durante las primeras semanas de 2015.

\section{Coberturas petroleras}

Las coberturas se refieren a la toma de una o varias posiciones de inversión (contratos de futuros, forward, swaps, opciones, índices, etc.) con el fin de compensar las posibles pérdidas y/o riesgo en que eventualmente incurre otra inversión; por tanto, el comportamiento de ambos debe estar correlacionado, ya sea de manera positiva o negativa. Operativamente, lo primero que se hace es determinar la relación que existe entre el activo a cubrir y el instrumento utilizado como cobertura (subyacente). Si ambos tienen una relación directa, entonces se cumple el siguiente principio: Cambio $\% \mathrm{~A}=\alpha *$ Cambio $\% \mathrm{~B}$, donde $\alpha$ es el coeficiente de cobertura o pendiente de la función, que mide el porcentaje de variación de $\mathrm{A}$ asociado a una variación de $1 \%$ de $\mathrm{B}$, e indica cuántas unidades de este instrumento deberán venderse o comprarse para respaldar el valor del activo cubierto. Los más usados son los contratos de futuros y los forward; ambos determinan el precio a futuro en que se llevará a cabo una compra o venta, aunque los primeros se realizan en los mercados regulados y los segundos lo hacen "sobre el mostrador" (mercados no regulados).

Por ejemplo, si la tesorería de una empresa compra acciones (A) por un valor de 40000 dólares de la compañía X, dedicada a la logística, e históricamente un aumento de $1 \%$ en el precio del combustible (PC) provoca una caída en el de las acciones del $0.7 \%(\alpha=-0.7)$, la así llamada ley de las probabilidades 
sugeriría establecer una función como la siguiente: $\Delta \mathrm{A}=-0.7 \Delta \mathrm{PC}$. Idealmente, la tesorería cubriría el riesgo asociado a la tenencia de las acciones comprando 28000 dólares (0.7 por 40 000) de futuros sobre el combustible. Ello implica que, en caso de que posteriormente suba el precio del energético, la pérdida de precio de las acciones se compensaría con la ganancia de los combustibles: de la función se deduce que un alza de $5 \%$ en el precio del combustible se anula con una reducción de $3.5 \%$ en el de las acciones $(\alpha=-0.7)$. Así, mientras la caída en el valor de las acciones supone una pérdida de 1400 dólares (3.5\% de 40 000) ésta se compensaría con una ganancia de 1400 dólares por el alza del combustible (5\% de 28 000). En el mundo real, más que eliminar el riesgo las coberturas sirven para minimizarlo.

Sobre la base expuesta, el gobierno de México ha adquirido, por lo menos desde 2002, coberturas para protegerse de las oscilaciones en el precio del petróleo, con cargo al Fondo de Estabilización de los Ingresos Petroleros (FEIP). Los costos acumulados de las primas entre dicho año y 2014 ascendieron a 99200 millones de pesos, equivalentes a unos 8000 millones de dólares a un tipo de cambio promedio para el periodo de 12.40 pesos por dólar.

\section{Efectos en las finanzas públicas}

En el caso del ejercicio 2015, a partir de octubre de 2014 las autoridades financieras se abocaron a la compra de opciones tipo 'put' (éstas dan al poseedor derecho, pero no obligación, de vender un activo a un precio predeterminado hasta una fecha específica) para la cobertura de $57.3 \%$ del crudo mexicano que se planeaba exportar en 2015 a un precio promedio de 76.4 d/b. El recurso subyacente fueron 228 millones de barriles de crudo mexicano que Pemex debe haber comprometido con el operador financiero al precio que se fijaba en el mercado de futuros durante la primera quincena de noviembre de 2014, muy cercano al que se aprecia para dicho lapso en la gráfica 1, presentada antes.

El costo de la prima se estableció en 773 millones de dólares, equivalentes 10467 millones de pesos de ese momento. El seguro se activa únicamente si en realidad durante 2015 el crudo registra un precio promedio inferior a 76.4 $\mathrm{d} / \mathrm{b}$, y esto no se sabe hasta que ya está muy avanzado el año. La última vez que se activó fue en 2009, año en que los precios promedio se situaron en 57.4 $\mathrm{d} / \mathrm{b}$, nivel inferior al de $70 \mathrm{~d} / \mathrm{b}$ promedio anual a que se contrató la cobertura.

La diferencia entre el precio contratado de la prima $(76.4 \mathrm{~d} / \mathrm{b})$ y el promedio estimado para 2015, con base en registros al primer mes del año (39.26 $\mathrm{d} / \mathrm{b}$ ), es de $-39.74 \mathrm{~d} / \mathrm{b}$. Aplicando esta cifra a los 228 millones de barriles asegurados se observa que el monto recuperado al final del año podría ser de 
Economía Informa núm. 391 marzo - abril• 2015

9061 millones de dólares. Transformados al tipo de cambio peso/dólar esperado de 14.50 , se llega a 131380 millones de pesos, equivalentes a $0.78 \%$ del PIB promedio de 2014 , y $0.72 \%$ si se le resta el costo de la prima.

En el cuadro 2 se hace el cálculo del efecto de la caída del precio del crudo en la parte no cubierta $(42.7 \%$ ) de las exportaciones petroleras (valores con signo negativo en millones de pesos de la parte 1). A éste se suman los beneficios que implica la reducción del precio de las gasolinas y diesel que por ley importa Pemex (valores con signo positivo en millones de pesos de la parte 2), sobre la base histórica de que éstos son en promedio 30\% superiores a los del crudo exportado. Como se observa en el renglón de ingresos netos, una vez calculado el efecto en el presupuesto por la caída del precio, se le agrega el costo de la prima, cantidad que se aplica a la parte de recursos no cubiertos y se llega a un total de 114102.4 millones de pesos, equivalentes a $0.67 \%$ del PIB.

\section{Cuadro 2}

\section{Efectos presupuestales del menor precio del crudo de exportación}

Proyecciones a partir de cifras observadas al primer mes del año

\begin{tabular}{|c|c|c|c|c|c|}
\hline & Presupuestado & Estimado & Dif (O-P) & $\begin{array}{c}\text { Sin } \\
\text { coberturas }\end{array}$ & $\%$ del PIB \\
\hline \multicolumn{6}{|l|}{ 1. Crudo } \\
\hline $\operatorname{Precio}(\mathrm{d} / \mathrm{b})$ & 79 & 39.26 & -39.74 & & \\
\hline Exportaciones (mbd) & 1090 & 1090 & 0 & & \\
\hline Ingresos en Mills. Dls. & 31430 & 15619 & -15811 & & \\
\hline Tipo cambio (pes/dol) & 13.4 & 14.5 & 1.1 & & \\
\hline Ingresos en Mills. pesos & 421162 & 226475.5 & -194686.5 & -83131.13 & -0.49 \\
\hline Costo cobertura & 0 & -10467 & -10467 & -10467 & -0.06 \\
\hline Ingresos netos Mills. pesos & 421162 & 216008.5 & -205153.5 & -93598.13 & -0.55 \\
\hline \multicolumn{6}{|l|}{ 2. Gasolinas y diesel } \\
\hline Precio (d/b) & 102.7 & 51 & -51.7 & & \\
\hline Importaciones (mdb) & 491 & 491 & 0 & & \\
\hline Egresos en Mills. Dls. & 18405.4 & 9140 & -9265.4 & Ahorros: & \\
\hline Egresos en Mills. pesos & -246632.4 & -132530 & 114102.4 & 114102.4 & 0.67 \\
\hline 3. saldo (I - E) & 667794.4 & 348538.5 & -319255.9 & 20504.26 & 0.1 \\
\hline
\end{tabular}

Principales supuestos: a) El precio del crudo observado al cierre del primer mes de 2015 se mantiene como promedio para el año, b) El precio de gasolinas y diesel importados se ubica $30 \%$ arriba del crudo exportado (promedio últimos años), c) Las exportaciones de crudo se mantienen constantes en su cifra presupuestada, mientras las importaciones de gasolinas y diesel se fijan al nivel observado en 2014 Fuentes: con base en cifras de Pemex, Indicadores Petroleros, Dic., 2014 y de SHCP, Presupuesto de Egresos 2015 e información sobre coberturas petroleras: protegen $228 \mathrm{mmb}$ a un precio de $76.4 \mathrm{~d} / \mathrm{b}(57.3 \%$ del valor de las exportaciones). 
Por tanto, el efecto neto del la caída de los precios del petróleo en el presupuesto de egresos se vuelve positivo en 20504.3 millones de pesos, $0.01 \%$ del piB. Es decir que dada la estructura de las exportaciones e importaciones petroleras de México, y habida cuenta de las coberturas petroleras, la baja de los precios en 2015 más que perjudicial podría ser benéfica para los ingresos de la SHCP.

El 30 de enero de 2015 la sHCP optó por aplicar un recorte al gasto público presupuestal del ejercicio en curso de 124300 millones de pesos. El monto equivale a $0.7 \%$ del PIB promedio estimado para 2015 (éste debe haberlo calculado aplicando al PIB nominal promedio de 2014 una inflación cercana a 4\% y un crecimiento real del producto de $2.7 \%$ ). Es evidente que el recorte es prácticamente igual a los recursos que posiblemente recuperará la SHCP de la cobertura: 131380.44 millones de pesos menos 10467 millones de costo de la prima $=120913.44$ millones de pesos, equivalentes a $0.71 \%$ del PIB. $^{2}$ ¿Por qué recortó un poco más del monto esperado de pérdida de recursos por concepto de exportaciones de crudo? ¿Por qué dejó de hacer mención de las coberturas petroleras? ¿Por qué nunca reconoció en sus cálculos los ahorros por el abaratamiento de las gasolinas y el diesel importados? ¿Por qué nunca dijo que en el balance neto el dinero recuperado de las coberturas (quizás a finales del año) será un ingreso adicional para las finanzas públicas que se sumaría al aumento de la recaudación tributaria gracias al avance de la reforma hacendaria?

Los mexicanos han aprendido que, cuando el gobierno aplica recortes en respuesta a reducciones en el precio del crudo mexicano de exportación, lo hace con cierta holgura, a fin de no tener que repetir la medida durante el año. De ahí la magnitud de los recortes de 1982, 1986, 1998, 2001 y 2009. En todos esos casos, la caída de los precios se asoció o bien a procesos de aguda desaceleración económica o bien de franca recesión. En 2015, por el contrario, la situación es otra: gracias al dinamismo de la economía estadounidense nadie habla de recesión en México; ni siquiera de contracción. Así que la única explicación que se puede dar al recorte es que, al ser 2015 año de elecciones, una parte de los recursos recuperados se van a ir a gasto corriente y otra parte a evitar que el déficit de las finanzas públicas frene su ascenso, para empezar a descender a partir de 2016 y acercarse a cero al final de la administración. Es esta segunda parte la que explica el beneplácito de las calificadoras

\footnotetext{
${ }^{2}$ El cálculo se efectúa multiplicando los 228 millones de crudo cubierto por el diferencial precio presupuestado - precio esperado de la mezcla de crudo para 2015 (39.74 d/b), lo que da 9060.72 millones de dólares. Al multiplicar estos por el tipo de cambio esperado de 14.50 pesos por dólar, se llega a los 131380.44 pesos. Al sustraerles el costo de la prima (10 467 millones de pesos), se obtienen los 120913.44 millones de pesos.
} 
internacionales y las organizaciones empresariales del país respecto a la austeridad de la sHCP.

Como se desprende de la gráfica 3, el esfuerzo de ajuste fiscal a partir de 2016 deberá ser muy grande, ya que un año después de la reforma energética de 2008, basada en leyes secundarias, que incluyó el relajamiento de la Ley Federal de Presupuesto y Responsabilidad Hacendaria en materia de mantenimiento de un déficit cero de las finanzas del sector púbico, las dos formas principales de desequilibrio fiscal se dispararon: el déficit del sector público pasó de 0\% en promedio con relación al PIB entre 2005 y 2008 a 2.3\% en 2009 y los RFSP subieron de $1.75 \%$ promedio a 5.3\% entre ambos lapsos. Para 2014 el primer coeficiente alcanzó el nivel más alto en lo que va del siglo, 3.6\%, y el segundo bajó a 4.2\%. Por supuesto el extraordinario salto de las dos cifras entre 2008 y 2009 se explica por la decisión del Congreso de convertir en deuda pública los Proyectos de Inversión con Impacto Diferido en el Gasto (PIDIREGAS) de Pemex. El mismo problema se observará en cuanto el gobierno federal asuma una parte de los pasivos pensionarios de Pemex y CFE, de acuerdo con lo establecido en las leyes secundarias de la reforma energética de 2013-2014.

\section{Gráfica 3}

Déficit y requerimientos financieros del sector público 2005-2014

Porcientos respecto al PIB

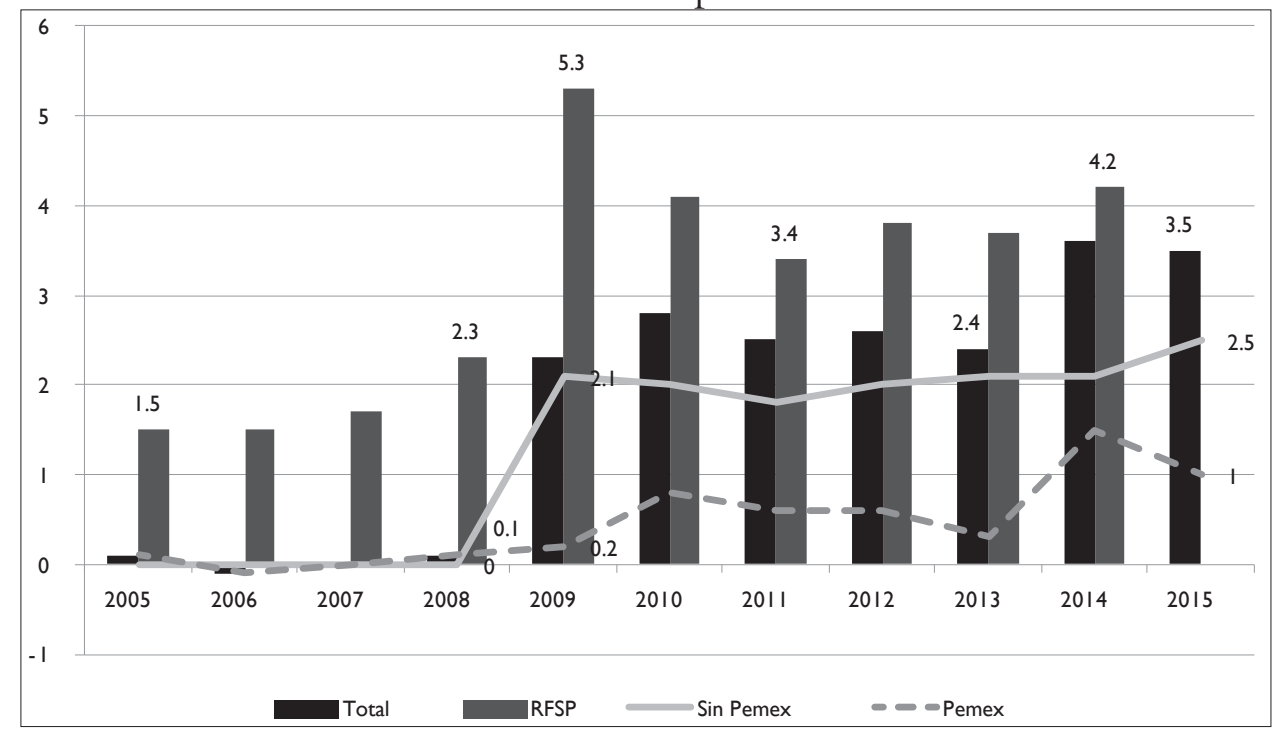

Fuente: con base en sHCP, Paquete Económico, diferentes años. 
Otro efecto de la reducción de los precios, aunado a la desaceleración permanente de la producción de hidrocarburos desde que el yacimiento de Cantarell tocó techo, a fines de 2004, debido a que la participación de Pemex en los ingresos tributarios del gobierno federal bajó de un promedio de $45 \%$ entre 2004-2007 a 33.2\% en 2013, 30.5\% en 2014 y posiblemente $27 \%$ en 2015.

Es importante recordar que todas las proyecciones económicas para 20152018 elaboradas durante el proceso de negociación de la reforma energética se habían hecho suponiendo ingresos de divisas, tan sólo para la rama de hidrocarburos, de entre 25000 y 50000 millones de dólares anuales, a las que se asociaba la creación de 500 mil empleos entre 2014 y 2018.

\section{Deuda externa}

Con una reforma hacendaria comprometida a no volver a aumentar los impuestos a los contribuyentes privados, la única opción del gobierno federal para mantener estable el déficit es la contención del gasto público. Desafortunadamente, varios de los proyectos que se cancelaron con el recorte anunciado por la SHCP resultaban cruciales para el crecimiento económico de corto y mediano plazos. En particular destacan los 62000 millones de pesos que se retiraron a Pemex, los 10000 millones a la Comisión Federal de Electricidad (CFE), el 33\% de los 11800 millones correspondientes a gasto de inversión que dejarán de ejercer las dependencias y entidades del sector público, así como la cancelación del tren rápido México-Querétaro y la postergación del proyecto tren transpeninsular Mérida-Punta Venado. No es exagerado afirmar que $70 \%$ del recorte es sobre el gasto de inversión, lo que sumado a la astringencia de IED hacia el sector energético implica que las medidas tendrán efectos no sólo anuales sino intertemporales.

Dadas las circunstancias descritas, a las que se suma el hecho de que el precio del petróleo podría no tener la recuperación deseada, cabe preguntarse ¿de dónde va a salir el dinero que permita mantener el gasto de inversión del sector público en un nivel compatible con un crecimiento razonable de la economía para el mediano plazo? En primer lugar, no existe seguridad de que en 2016 empiece a fluir la IED al sector energético y al resto de la economía. En segundo lugar, y debido al pacto fiscal autoimpuesto por la sHCP, los ingresos tributarios seguirán atados a los niveles establecidos en diciembre de 2013 y su crecimiento dependerá sobre todo del aumento de la base gravable y de la capacidad fiscalizadora del SAT. En tercer lugar, ya no es posible recurrir a 
coberturas para protegerse de una nueva caída de los precios del petróleo, en virtud de que los futuros de dicho recurso evidentemente estarán deprimidos durante todo 2015. Y en cuarto lugar, el alza esperada de las tasas de interés de EU, que tanto ha enfatizado el Banco de México, no sólo podría deteriorar el flujo neto de inversión de cartera a México, sino que encarecerá el servicio de la deuda externa, pública, privada y de la banca comercial, que como muestra el cuadro 3 ascendió en 2014 a 266317 millones de dólares, cifra equivalente a $20 \%$ del PIB.

Sorprendentemente, la deuda que más rápido crece es la del sector privado, 9.7\% promedio anual desde 2010 (la total lo hace a un ritmo de 8.2\%). Si se agrega a ésta la de la banca comercial, ambas representan $43.3 \%$ del saldo total. Por el lado del sector público, es evidente que la entidad más endeudada es Pemex, con pasivos cercanos a 70000 millones de dólares, los cuales crecen a la velocidad más alta de casi toda la economía, gracias a que se trata de una entidad del Estado que además garantiza con hidrocarburos sus pasivos financieros.

\section{Cuadro 3}

Deuda externa total de México Millones de dólares

\begin{tabular}{|c|c|c|c|c|c|}
\hline Concepto & 2010 & 2011 & 2012 & 2013 & $2014 p$ \\
\hline Total & 190143 & 201344 & 213410 & 236821 & 266317 \\
\hline Sector público & 110428 & 116420 & 125726 & 134436 & 151042 \\
\hline Sector privado & 64707 & 68650 & 72025 & 86410 & 93819 \\
\hline Banca comercial & 15008 & 16273 & 15659 & 15975 & 21456 \\
\hline
\end{tabular}

p: preliminar.

Fuente: con información de la SHCP.

\section{Conclusiones}

La caída de los precios del petróleo de 2014-2015 tendrá varios efectos en la economía mexicana. En primer lugar destacan los asociados a la balanza de pagos, cuya cuenta comercial seguramente registrará un déficit considerable en 2015, sobre todo porque éste se asociaría a un saldo negativo de la balanza petrolera, el primero desde principios de los años setenta del siglo xx, y a un crecimiento de la economía cercano a 2.5\%. En principio, el déficit comercial deberá conducir a un mayor déficit de cuenta corriente, el cual tendría que financiarse con recursos provenientes de la balanza de capital y financiera. 
Pero como parece estar previendo el Banco de México, el aumento esperado de la tasa de interés en Eu reducirá el flujo neto de capital financiero al país y encarecerá el servicio de la deuda externa, cuyo saldo en 2014 representó 20\% del PIB, con un endeudamiento per capita de 219 dólares, el más alto de la historia. Asimismo, al ser 2015 un año de transición para la industria petrolera, se postergará hasta por lo menos 2016 la IED en hidrocarburos.

En cuanto a la dinámica económica, al centrarse la mayor parte de la reducción de $0.7 \%$ del gasto público programado para 2015 en el sector energético y en proyectos de inversión comprometidos desde el inicio de la administración Peña, la inversión pública perderá la oportunidad de consolidar cierta capacidad de arrastre sobre la privada. Así, la economía podría perder en 2015 hasta 0.5 puntos porcentuales del nivel de crecimiento que habría alcanzado si no se hubieran cancelado los proyectos, con efectos rezagados para los años posteriores. Esto desvanecerá completamente el objetivo de que en el presente sexenio se alcance un promedio de crecimiento económico de al menos el doble de los dos anteriores (2.4\%). Si en términos per cápita se llega a 2\%, será un verdadero logro.

En un contexto de reducción de los precios internacionales del petróleo y de contracción de la inversión, el peso de la industria de hidrocarburos en el PIB deberá bajar de 7\% en 2012 a 6.5\% en 2015. En cuanto a la contribuciones de la industria en los ingresos tributarios del gobierno federal seguirá su tendencia descendente: de $45 \%$ entre 2004 y 2006 a 33.2\% en $2013,30.5 \%$ en 2014 y posiblemente $27 \%$ en 2015 .

La compra de coberturas petroleras pudo haberse utilizado, en una primera instancia, y combinada con los ahorros que generan las importaciones de gasolinas y diesel, para evitar el recorte al gasto; en una segunda instancia, podrían servir para reforzar los ingresos y reducir las presiones sobre el déficit, propias de un año electoral. Empero, la SHCP no quiso esperar que se acercara el final del año para recibir los recursos, y mientras acordar diferimientos de pagos con los proveedores del sector público. Tampoco parece haber tomado en cuenta los efectos de la reforma hacendaria, por la que subió 1.3 puntos porcentuales la participación de los ingresos tributarios no petroleros en el PIB (15.2\% en 2013; 16.5\% en 2014), ni los nuevos mecanismos recaudatorios del SAT y la expectativa de que para final del sexenio llegue a 3\% el aumento de este estrato recaudatorio.

Por tanto, debe interpretarse que el gobierno federal ha encontrado en la vorágine del mercado petrolero internacional un mecanismo para no aumentar en un año de elecciones el déficit del sector público, que en 2014 alcanzó su nivel más alto del siglo, 3.6\% del PIB (4.2\% en términos de los RFSP). Aunque esto en sí mismo constituye una buena señal, ya que habla de disciplina 
fiscal, el mensaje que se manda no es compatible, ya que se renuncia al liderazgo en materia de inversión, y no queda claro que la iniciativa privada vaya a tomar la estafeta, particularmente porque como lo ha hecho explícito no está dispuesta a invertir si se sostiene la actual política tributaria. A esto se suman los temores que se han propalado desde la administración pública respecto a la duración del choque petrolero, la volatilidad de la paridad cambiaria y el inminente aumento de la tasa de interés de EU, sin hacer mención a los avances en materia recaudatoria y al empleo de instrumentos que ayudan en mucho a mitigar el efecto de la caída de los precios internacionales del crudo.

Más que crear la imagen de una economía mexicana ensombrecida y de prometer, como se ha hecho desde los años ochenta, que en 2016 por fin se pondrá en operación un sistema de presupuestación base cero, lo que seguramente implicará la continuación del proceso de reducción del gasto público iniciado en 2015 al que deberán incluirse tanto los tres poderes como los órganos no regulados, el mandato ciudadano es saber exactamente a dónde van los recursos públicos, como se manejan, si esto se hace buscando su optimización y si se puede tener la seguridad de que los amplios conceptos de transparencia y rendición de cuentas son sinónimos de ética y eficiencia.

\section{Bibliografía}

British Petroleum (вр) (2014), вP Statistical Review of World Energy, junio, www. bp.com consultado en febrero de 2015.

Instituto Nacional de Estadística y Geografía (INEGI), Banco de Información Económica, www.inegi.org.mx consultado en enero de 2015.

Secretaría de Hacienda y Crédito Público (SHCP) (2015), Estadísticas Oportunas de Finanzas Públicas, www.shcp.gob.mx consultado en enero de 2015

(2015), Paquete Económico 2015 y años anteriores, www.shcp.gob.mx consultado en enero de 2015

Peña Nieto, Enrique (2011), México, la Gran Esperanza. Un Estado Eficaz para una Democracia de Resultados, México: Grijalbo Mondadori.

Petróleos Mexicanos (Pemex) (2014), Indicadores Petroleros, www.pemex.com.mx consultado en enero de 2015.

(2014) Anuario Estadístico 2014 y años anteriores, www.pemex.com.mx consultado en enero de 2015.

Presidencia de la República (2012), Pacto por México, México, diciembre 2.

Ugalde, Luis Carlos (coordinador) (2014), La Negociación Política del Presupuesto en México 1997-2012, México: Integralia. 